\section{Cardioimmunology of arrhythmias: the role of autoimmune and inflammatory cardiac channelopathies}

\author{
Pietro Enea Lazzerini(D, Franco Laghi-Pasini, Mohamed Boutjdir and \\ Pier Leopoldo Capecchi
}

We have read with great interest the excellent review on cardioimmunology by Filip K. Swirski \& Matthias Nahrendorf (Cardioimmunology: the immune system in cardiac homeostasis and disease. Nat. Rev. Immunol. 18, 733-744 (2018)) ${ }^{1}$. The authors extensively discussed the role of immune cells in normal and diseased heart, specifically in myocardial infarction, myocarditis and endocarditis, heart failure and rhythm disorders ${ }^{1}$. Regarding rhythm disorders, they speculated that the immune system could contribute to arrhythmias through two mechanisms - a crosstalk between immune cells and fibroblasts and/or myocytes, leading to insulating fibrosis, or a direct participation of leukocytes (macrophages) in the electrical regulation of conducting cells, by interacting through connexin 43 (CX43)-containing gap junctions ${ }^{1}$.

However, the authors substantially disregarded a third important mechanism of arrhythmias in this new field of

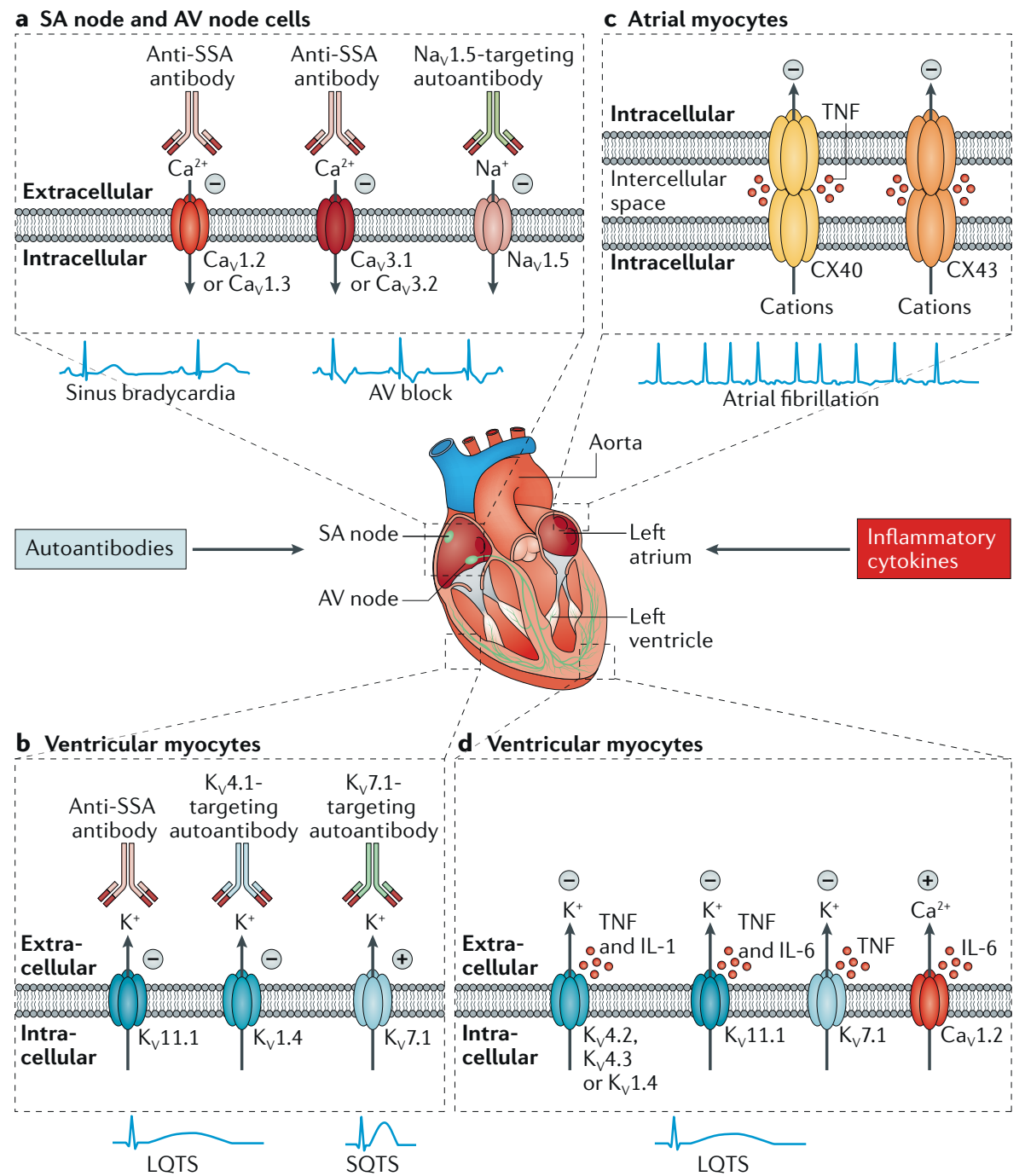

immuno-cardiac electrophysiology. In fact, accumulating data indicate that the immune system can promote cardiac arrhythmias by means of autoantibodies and/or inflammatory cytokines that directly affect the function of specific ion channels on the surface of cardiomyocytes ${ }^{2-4}$.

Several autoantibodies have been described that target cardiac $\mathrm{Ca}^{2+}, \mathrm{K}^{+}$or $\mathrm{Na}^{+}$ channels and that have arrhythmogenic effects in the absence of evident histological changes in the heart; these are known as autoimmune cardiac channelopathies ${ }^{2,4}$. Indeed, it is well recognized that antiSjögren's-syndrome-related antigen A (anti-SSA) antibodies (also known as antiRo/SSA antibodies) can cross react with the pore region of both L-type $\mathrm{Ca}^{2+}$ channels $\left(\mathrm{Ca}_{\mathrm{V}} 1.2\right.$ and $\left.\mathrm{Ca}_{\mathrm{v}} 1.3\right)$ and T-type $\mathrm{Ca}^{2+}$ channels $\left(\mathrm{Ca}_{\mathrm{v}} 3.1\right.$ and $\left.\mathrm{Ca}_{\mathrm{v}} 3.2\right)$. By inhibiting the related $\mathrm{Ca}^{2+}$ currents, these antibodies promote conduction disturbances, such as sinus bradycardia and atrioventricular (AV) block $^{5,6}$ (FIG. 1 a). Similar clinical consequences were also shown for autoantibodies recognizing the extracellular loop of domain

Fig. 1 | Autoimmune and inflammatory cardiac channelopathies and arrhythmias: molecular basis. Autoantibodies and inflammatory cytokines can modulate the function of cardiac ion channels and promote arrhythmias. a | Bradyarrhythmias and conduction disturbances can be induced by anti-Sjögren's-syndrome-related antigen A (anti-SSA) antibodies (also known as anti-Ro/SSA antibodies), which target the L-type and/or T-type $\mathrm{Ca}^{2+}$ channels and inhibit the related currents, or by autoantibodies targeting the $\mathrm{Na}_{\mathrm{v}} 1.5 \mathrm{Na}^{+}$channel, which inhibit the $\mathrm{Na}^{+}$current, in cells of the sinoatrial (SA) node and atrioventricular (AV) node. b | Long-QT syndrome (LQTS) can be induced by anti-SSA antibodies, which target the $\mathrm{K}_{\mathrm{v}} 11.1 \mathrm{~K}^{+}$channel (also known as $\mathrm{hERG}$ ) and inhibit the rapidly activating repolarizing component of the delayed rectifier $\mathrm{K}^{+}$current, or by autoantibodies targeting the $\mathrm{K}_{\mathrm{v}} 1.4 \mathrm{~K}^{+}$channel, which might inhibit the transient outward $\mathrm{K}^{+}$current in ventricular myocytes. Short-QT syndrome (SQTS) can be induced by autoantibodies targeting the $\mathrm{K}_{\mathrm{v}} 7.1 \mathrm{~K}^{+}$channel, which increase the slowly activating repolarizing component of the delayed rectifier $\mathrm{K}^{+}$current in ventricular myocytes. c Atrial fibrillation can be induced by tumour necrosis factor (TNF), which impairs the expression and/or distribution of connexin 40 (CX40) and CX43 and inhibits the function of gap junctions in atrial myocytes. $\mathbf{d}$ | LQTS can be induced by TNF, which targets $\mathrm{K}_{\mathrm{v}} 4.2, \mathrm{~K}_{\mathrm{v}} 4.3, \mathrm{~K}_{\mathrm{v}} 11.1$ and $\mathrm{K}_{\mathrm{v}} 7.1 \mathrm{~K}^{+}$ channels and inhibits the respective currents; by IL-1, which inhibits the transient outward $\mathrm{K}^{+}$ current; or by IL- 6 , which targets the L-type $\mathrm{Ca}^{2+}$ channel ( $\left.\mathrm{Ca}_{\mathrm{v}} 1.2\right)$ and increases the L-type $\mathrm{Ca}^{2+}$ current, in ventricular myocytes. For simplicity, IL-6 and TNF receptors are not shown in panel d. 
I S5-S6 of the $\mathrm{Na}_{\mathrm{v}} 1.5 \mathrm{Na}^{+}$channel7. These antibodies, which can be detected in patients with idiopathic $\mathrm{AV}$ block, inhibit $\mathrm{Na}^{+}$ currents and induce conduction disturbances in experimental models 7 .

Other autoantibodies that target ion channels can affect the action potential duration (APD) of ventricular myocytes, leading to long-QT syndrome (LQTS) or short-QT syndrome (SQTS) and associated malignant arrhythmias ${ }^{2}$. LQTS can be induced by anti-SSA antibodies, which inhibit the rapidly activating repolarizing $\mathrm{K}^{+}$current by targeting the extracellular pore loop of the $\mathrm{K}_{\mathrm{v}} 11.1 \mathrm{~K}^{+}$channel (also known as hERG) ${ }^{8,9}$, as well as by autoantibodies targeting $\mathrm{K}_{\mathrm{V}} 1.4$ $\mathrm{K}^{+}$channels, possibly through blockade of the transient outward $\mathrm{K}^{+}$current ${ }^{10}$. Conversely, agonist-like autoantibodies targeting $\mathrm{K}_{\mathrm{v}} 7.1 \mathrm{~K}^{+}$ channels that enhance the slowly activating repolarizing $\mathrm{K}^{+}$current were associated with SQTS $^{11}$ (FIG. 1 b).

Moreover, inflammatory cytokines - in particular, tumour necrosis factor (TNF), IL-1 and IL-6 - can be arrhythmogenic by directly affecting the function of cardiac ion channels; these are known as inflammatory cardiac channelopathies ${ }^{4}$. Specifically, it has been shown that TNF induces dysfunction of gap junctions in atrial myocytes through impaired expression and/or distribution of CX40 and CX43 and that these changes promote atrial fibrillation by favouring a slow and heterogeneous conduction in the atria $^{12}$ (FIG. 1c). In addition, cytokines can favour the development of LQTS by decreasing specific cardiac $\mathrm{K}^{+}$currents and/or increasing L-type $\mathrm{Ca}^{2+}$ currents ${ }^{3,4}$. TNF inhibits transient outward, rapidly activating repolarizing and slowly activating repolarizing $\mathrm{K}^{+}$currents as a result of the downregulation of channel expression and/or alterations in channel-gating kinetics, which are also associated with prolongation of the APD and/or QT interval ${ }^{4,13}$. Similar effects are mediated by IL-1, which reduces the transient outward $\mathrm{K}^{+}$current $^{14}$, and by IL-6, which enhances the L-type $\mathrm{Ca}^{2+}$ current through $\mathrm{Ca}_{\mathrm{v}} 1.2$ phosphorylation ${ }^{15}$ and inhibits the rapidly activating repolarizing $\mathrm{K}^{+}$current through a pathway involving the IL-6 receptor and Janus kinase ${ }^{16}$ (FIG. 1 d).

In terms of translational medicine, emphasizing the role of autoimmune and inflammatory cardiac channelopathies in arrhythmogenesis may lead to innovative anti-arrhythmic therapies based on the targeted modulation of the immuneinflammatory system, such as cytokinetargeting monoclonal antibodies or short decoy peptides that divert autoantibodies from their binding sites on ion channels.

There is a reply to this letter by Swirski, F. K. \& Nahrendorf, M. Nat. Rev. Immunol. https:// doi.org/10.1038/s41577-018-0099-y (2018).

Pietro Enea Lazzerini (iD) ${ }^{1}$, Franco Laghi-Pasini ${ }^{1,4}$, Mohamed Boutjdir, ${ }^{2,3,4}$ and Pier Leopoldo Capecchir, ${ }^{1}$ Department of Medical Sciences, Surgery and Neurosciences, University of Siena, Siena, Italy. 2VA New York Harbor Healthcare System, SUNY Downstate Medical Center, New York, NY, USA. ${ }^{3}$ NYU School of Medicine, New York, NY, USA. ${ }^{4}$ These authors contributed equally: Franco LaghiPasini, Mohamed Boutjdir, Pier Leopoldo Capecchi. *e-mail: lazzerini7@unisi.it https://doi.org/10.1038/s41577-018-0098-z

1. Swirski, F. K. \& Nahrendorf, M. Cardioimmunology: the immune system in cardiac homeostasis and disease. Nat. Rev. Immunol. 18, 733-744 (2018).

2. Lazzerini, P. E. et al. Autoimmune channelopathies as a novel mechanism in cardiac arrhythmias. Nat. Rev. Cardiol. 14, 521-535 (2017).
3. Lazzerini, P. E., Capecchi, P. L. \& Laghi-Pasini, F Systemic inflammation and arrhythmic risk: lessons from rheumatoid arthritis. Eur. Heart J. 38 1717-1727 (2017).

4. Lazzerini, P. E. et al. Emerging arrhythmic risk of autoimmune and inflammatory cardiac channelopathies. J. Am. Heart Assoc. 7, e010595 (2018).

5. Xiao, G. Q., Hu, K. \& Boutjdir, M. Direct inhibition of expressed cardiac L- and T-type calcium channels by IgC from mothers whose children have congenital heart block. Circulation 103 1599-1604 (2001)

6. Karnabi, E. et al. Congenital heart block: identification of autoantibody binding site on the extracellular loop (domain I, S5-S6) of $\alpha_{10}$ L-type Ca channel. J. Autoimmun 34, 80-86 (2010).

7. Korkmaz, S. et al. Provocation of an autoimmune response to cardiac voltage-gated sodium channel $\mathrm{Na}_{\mathrm{v}} 1.5$ induces cardiac conduction defects in rats. J. Am. Coll. Cardiol. 62, 340-349 (2013).

8. Yue, Y. et al. Pathogenesis of the novel autoimmune-associated long-QT syndrome. Circulation 132, 230-240 (2015).

9. Lazzerini, P. E. et al. Arrhythmogenicity of anti-Ro/SSA antibodies in patients with torsades de pointes. Circ. Arrhythm. Electrophysiol 9, e003419 (2016).

10. Suzuki, S. et al. Cardiac involvements in myasthenia gravis associated with anti- $\mathrm{K}_{\mathrm{v}} 1.4$ antibodies. Eur. J. Neurol. 21, 223-230 (2014).

11. $\mathrm{Li}$, J. et al. Anti-KCNQ1 $\mathrm{K}^{+}$channel autoantibodies increase IKs current and are associated with OT interval shortening in dilated cardiomyopathy. Cardiovasc. Res. 98, 496-503 (2013).

12. Sawaya, S. E. et al. Downregulation of connexin 40 and increased prevalence of atrial arrhythmias in transgenic mice with cardiacrestricted overexpression of tumor necrosis factor. Am. J. Physiol. Heart Circ. Physiol. 292 H1561-H1567 (2007).

13. Wang, J. et al. Impairment of HERG $\mathrm{K}^{+}$channel function by tumor necrosis factor-alpha: role of reactive oxygen species as a mediator. J. Biol. Chem 279, 13289-13292 (2004).

14. Monnerat, G. et al. Macrophage-dependent IL-1 $\beta$ production induces cardiac arrhythmias in diabetic mice. Nat. Commun. 7, 13344 (2016)

15. Hagiwara, Y. et al. SHP2-mediated signaling cascade through gp 130 is essential for LIF-dependent I CaL, $\left[\mathrm{Ca}^{2+}\right] \mathrm{i}$ transient, and APD increase in cardiomyocytes. J. Mol. Cell. Cardiol. 43, 710-716 (2007).

16. Aromolaran A S et al Interleukin-6 inhibition of hERG underlies risk for acquired long OT in cardiac and systemic inflammation. PLoS One. 13(12), e0208321 (2018)

Competing interests

The authors declare no competing interests. 